\title{
Coexistence and crossover phenomena in a Fermi-like model of particles in counterflowing streams
}

\author{
Eduardo Velasco Stock $\odot$ and Roberto da Silva $\odot$ \\ Instituto de Física, Universidade Federal do Rio Grande do Sul, UFRGS, Porto Alegre-RS, 91501-970, Brazil
}

(Received 28 February 2020; revised 19 June 2020; accepted 4 August 2020; published 26 August 2020)

\begin{abstract}
In this work, we propose a two-dimensional extension of a previously defined one-dimensional version of a model of particles in counterflowing streams, which considers an adapted Fermi-Dirac distribution to describe the transition probabilities. In this modified and extended version of the model, we consider that only particles of different species can interact, and they hop through the cells of a two-dimensional rectangular lattice with probabilities taking into account diffusive and scattering aspects. We show that for a sufficiently low level of randomness $(\alpha \geqslant 10)$, the system can relax to a mobile self-organized steady state of counterflow (lane formation) or to an immobile state (clogging) if the system has an average density near a certain crossover value $\left(\rho_{c}\right)$. We also show that in the case of imbalance between the species, we can simultaneously have three different situations for the same density value set: (i) an immobile phase, (ii) a mobile pattern organized by lanes, and (iii) a profile with mobility but without lane formation, which essentially is the coexistence of situations (i) and (ii). All of our results were obtained by performing Monte Carlo simulations.
\end{abstract}

DOI: 10.1103/PhysRevE.102.022139

\section{INTRODUCTION}

The statistical mechanics of self-driven diffusive systems has great importance in the context of nonequilibrium phenomena [1]. In particular, counterflowing streams of particles [2-6] can lead to interesting patterns, such as those presented by studies with charged colloids [7-11], for example. Exactly as pedestrians organize their motion to make that system flow $[12,13]$, particles without a "natural intelligence," self-propelled or simply oriented by a field, sometimes can organize their motion by lanes (see, for example, Refs. $[14,15,16])$. However, exclusion effects seem to bring clogging/jamming [17] phenomena [18,19], although the formation of condensates is not mandatorily observed only in counterflowing systems [20].

Recently, we have developed a model with exclusion based on Fermi-Dirac distribution [18] from an extension of a simple model without exclusion previously defined in [3], which was loosely based on the concept of clannish random walks developed by Montroll and West [21]. The easiness and coverage of this extended model is related to the manipulation of a single parameter that can model systems going from hard-body systems with exclusion up to systems that are completely random and without exclusion effects. We have previously shown that such systems have an interesting transition from a clogging phase to a mobile phase, which we have described with many order parameters such as mobility, the Gini coefficient, etc., over different situations [19].

The extension of this Fermi model for the counterflowing streams of particles in two dimensions has not yet been studied, and it deserves our attention. It is known that models in counterflowing system of particles in two dimensions present interesting lane patterns. For example, a lattice model for oppositely driven binary particles with purely repulsive interactions was investigated on a square lattice presenting such an effect [22]. On the other hand, two-dimensional directed random walks in counterflow can also present such phenomena [5].

In this paper, we propose a two-dimensional version of the Fermi model to describe the counterflowing streams of particles on a lattice. We show that the system can present metastable events between a clogging and a lane phase, which, to the best of our knowledge, was never studied in systems with counterflowing streams of particles. Thus in this paper, we qualitatively and quantitatively explore this phenomenon in detail.

Our paper is organized as follows: In the next section, we present one of the possible formulations (the more appropriate one in our opinion) of the modeling in two dimensions. We show that renormalization is required for $d=2$, but that naturally recovers $d=1$ previously studied by us in our first contributions about this topic [18,19]. After that, we present our results in Sec. III. Finally, a few conclusions and summaries are presented in Sec. IV.

\section{THE MODEL IN TWO DIMENSIONS}

We start this section by defining our scenario: a rectangular system of $V=L_{x} L_{y}$ cells with periodic boundary conditions (toroidal lattice) where $N$ particles of two species, namely $A$ and $B$, can move. Each cell has a maximum occupation level denoted by $\sigma_{\max }$. Our particles are able to hop only to their neighboring cells where particles of species $A$ tend to move to the $+x$ direction (along the toroid) while particles of species $B$ tend to move to the $-x$ direction, exactly like the effect of an electric field longitudinally applied, considering that particles of species $A$ are oppositely charged to particles of species $B$. In our model, particles only interact with particles 
of the opposite type. Thus a particle of species $A$ that occupies the cell $(x, y)=(i \epsilon, j \epsilon)$ hops to its neighboring cell $(x, y)=$ $(i \epsilon+\epsilon, j \epsilon)$ at the instant $t=\tau l$ according to a rate that follows the Fermi-like distribution of probability given by

$$
\omega_{(i, j) \rightarrow(i+1, j)}^{(l)}=\frac{1}{1+e^{\alpha\left(B_{i+1, j}^{(l)}-\sigma_{\max }\right)}},
$$

where $B_{i+1, j}^{(l)}$ is the number of particles of the species $B$ in the cell, $\alpha$ is the parameter that controls the randomness of the dynamics, $\epsilon$ is the step length (linear dimension of the cells), and $\tau$ is the time spent to perform the transition.

We define the rate of the particles to move along the $y$ direction as a product of two factors: Scattering and diffusion. The scattering factor is defined as the complement of the rate of a particle to move in the direction of the toroid, i.e., the complement of Eq. (1). In a similar way, the diffusion factor consists of the rate of a particle entering the neighboring cell in the $y$-direction, which depends on the level of occupation of the target cell. If we only consider the diffusive factor as the "lateral" rates, the model would be able to be reduced to a set of single-lane systems because the dynamics along the two perpendicular axes would only be indirectly coupled (see, for example, [23]).

By this logic, we describe the rate at which particle $A$ moves to a cell in the transversal direction of the toroid as

$$
\begin{aligned}
\omega_{(i, j) \rightarrow(i, j+1)}^{(l)} & =\left[1-\frac{1}{\left.1+e^{\alpha\left(B_{i+1, j}^{(l)}-\sigma_{\max }\right.}\right)}\right] \cdot \frac{1}{\left.1+e^{\alpha\left(B_{i, j+1}^{(l)}-\sigma_{\max }\right.}\right)} \\
& =\frac{e^{\alpha\left(B_{i+1, j}^{(l)}-\sigma_{\max }\right)}\left(1+e^{\alpha\left(B_{i, j+1}^{(l)}-\sigma_{\max }\right)}\right)^{-1}}{\left(1+e^{\alpha\left(B_{i+1, j}^{(l)}-\sigma_{\max }\right)}\right)} .
\end{aligned}
$$

And similarly,

$$
\omega_{(i, j) \rightarrow(i, j-1)}^{(l)}=\frac{e^{\alpha\left(B_{i+1, j}^{(l)}-\sigma_{\max }\right)}\left(1+e^{\alpha\left(B_{i, j-1}^{(l)}-\sigma_{\max }\right)}\right)^{-1}}{\left(1+e^{\alpha\left(B_{i+1, j}^{(l)}-\sigma_{\max }\right)}\right)},
$$

where the term inside the square brackets corresponds to the scattering factor, and the term outside the square brackets corresponds to the factor of diffusion.

At this point, we are able to define the normalization factor:

$$
Z_{(i, j)}^{(l)}= \begin{cases}\Omega_{(i, j)}^{(l)} & \text { if } \Omega_{(i, j)}^{(l)} \geqslant 1, \\ 1 & \text { otherwise }\end{cases}
$$

where $\Omega_{(i, j)}^{(l)}=\sum_{\langle i, j\rangle} \omega_{(i, j) \rightarrow\left(i^{\prime}, j^{\prime}\right)}^{(l)}$ is the total rate of particles that come out of the cell, and here $\left\langle i^{\prime}, j^{\prime}\right\rangle$ denotes that the sum is taken over the nearest-neighbor cells toward which the movement is allowed. Thus using the normalization, we now have the transition probabilities defined as

$$
\begin{aligned}
& p_{(i, j) \rightarrow(i+1, j)}^{(l)}=\frac{1}{Z_{(i, j)}^{(l)}} \omega_{(i, j) \rightarrow(i+1, j)}^{(l)}, \\
& p_{(i, j) \rightarrow(i, j \pm 1)}^{(l)}=\frac{1}{Z_{(i, j)}^{(l)}} \omega_{(i, j) \rightarrow(i, j \pm 1)}^{(l)} .
\end{aligned}
$$

However, to keep the similarities with the original model, the particles also have to have the chance of remaining in their cells. So we also define the probability using

$$
p_{(i, j) \rightarrow(i, j)}^{(l)}=\left(1-\Omega_{(i, j)}^{(l)}\right) \Theta\left(1-\Omega_{(i, j)}^{(l)}\right),
$$

where $\Theta(\xi)$ is the step function:

$$
\Theta(\xi)=\left\{\begin{array}{lc}
1 & \text { if } \xi>0 \\
0 & \text { otherwise. }
\end{array}\right.
$$

By symmetry, a particle of species $B$ in the cell $(x, y)=$ $\epsilon(i, j)$ hops to its neighboring cell $(x, y)=(i \epsilon-\epsilon, j \epsilon)$ at the instant $t=\tau l$ according to the rate

$$
\omega_{(i, j) \rightarrow(i-1, j)}^{(l)}=\frac{1}{1+e^{\alpha\left(A_{i-1, j}^{(l)}-\sigma_{\max }\right)}},
$$

and the transition rate from $(x, y)=\epsilon(i, j)$ to $(x, y)=$ $(i \epsilon,(j \pm 1) \epsilon)$ is

$$
\omega_{(i, j) \rightarrow(i, j \pm 1)}^{(l)}=\frac{\left.e^{\alpha\left(A_{i-1, j}^{(l)}-\sigma_{\max }\right.}\right)\left(1+e^{\alpha\left(A_{i, j \pm 1}^{(l)}-\sigma_{\max }\right)}\right)^{-1}}{\left(1+e^{\alpha\left(A_{i-1, j}^{(l)}-\sigma_{\max }\right)}\right)} .
$$

By building $Z_{(i, j)}^{(l)}$, one similarly obtains the transition probabilities calculated for the particles $B$.

The main parameter of the adapted Fermi-like distribution in our model is $\alpha$ once it controls the randomness of the dynamics that can range from a purely random scenario to a deterministic case in which the relation between the cell's occupation and the limitation factor $\left(\sigma_{\max }\right)$ is paramount. In the context of counterflowing particle dynamics, $\alpha$ can be associated with the magnitude of an external field applied to oppositely charged particles.

We can understand the influence of $\alpha$ on the dynamics by focusing on the two extreme situations: (i) $\alpha \rightarrow 0$ and (ii) $\alpha \rightarrow \infty$. In the case (i) $(\alpha \rightarrow 0)$ the dynamics is random with particles $A(B)$ moving cell by cell with probability $1 / 2$ toward the $+(-) x$ direction if the particle is from the $A(B)$ species and with $1 / 4$ to the $\pm y$ direction. It is easy to notice that there is no chance that particles will stay in their cells. On the other hand, in the contrasting situation (ii) $(\alpha \rightarrow \infty)$, the system behaves with deterministic dynamics like the lattice gases, and the set of possible configurations of occupation of the surrounding cells will define with certainty the movement of all particles except for the particular case in which the target cell happens to have $\sigma=\sigma_{\max }$. In that case, a local random decision is made as a result of $p=1 / 2$ or $1 / 4$ depending on the direction of the occupied cell, although we do not expect it to substantially alter the bulk behavior of the system. Moreover, it is important to mention that we have a notorious computational advantage in using this model, since we are not obliged to check by brutal force if cells satisfy the occupation limit $\sigma_{\max }$ like systems with nearest-neighbor exclusion [24,25].

To describe the state of the dynamics, we define the mobility, denoted as $M(t)=\frac{1}{N} \sum_{i}^{N} \xi_{i}(t)$, where $\xi_{i}(t)$ is a binary parameter that assumes the value 1 when a particle moves in the $x$ direction, and the value 0 otherwise. The mobility stands as a current of particles or simply the fraction of the particles that perform a step along its specie's drift direction. When jamming/clog occurs the mobility drops to zero. While when an opposite scenario occurs like particle's self-organization in 
lane formation, the mobility will tend to one as a result of the optimal flow. To capture the lane-organized state we define the order parameter in the $x$-direction, or simply " $x$-order parameter," denoted as $\Phi_{x}^{l} \equiv \frac{1}{N} \sum_{k=1}^{L y}\left|A_{k}^{l}-B_{k}^{l}\right|$, where $A_{k}^{l} \equiv$ $\sum_{j=1}^{L x} A_{j, k}^{l}$ and $B_{k}^{l} \equiv \sum_{j=1}^{L x} B_{j, k}^{l}$ are the marginal sum over the $j$ variable (cells along the $y$-direction) of the number of particles of each species. From the definition of $\Phi_{x}^{l}$ we see that it will tend to 1 when the system is in a lane-organized state along the toroid, which represents an optimal state of flow due to the lack of particles moving in the opposite direction.

It is only possible to calculate the mobility parameter when performing MC simulations, differently from $\Phi_{x}$, which depends only on the spatial concentration of particles and can also be measured when one integrates the recurrence relations that emerge from a mean-field approximation of Eqs. (1)-(3) for species $A$ as well as it can be done for species $B$, similarly to what was done in Ref. [5].

We implemented standard MC simulations. For that, one uses an asynchronous updating method: First, a particle is drawn at random among $N$ possible choices. Thus, according to the transition rules previously explained, one updates the position of this particle. Sequentially, another particle is picked up randomly and the same procedure is successively repeated. One MC step consists of $N$ of these procedures (random sequential updates), where the same particle can possibly be chosen more than once in the same MC step. In all of our results, we use $N_{A}=N_{B}=N / 2$. One uses a uniformly random spatial distribution of the particles, and amounts used in this paper, such as $M(t)$ or $\Phi(t)$, were computed for each $t$ th MC-step; other variables that will be presented were averaged over $N_{\text {run }}=100000 / N+$ 10000 different runs unless specified otherwise. Each run corresponds to a different time series (different seeds) of the evolution that reaches the steady state in the experiment. This adaptive sampling is used to keep a reasonable number of runs in the case of diluted lattices.

A detailed study of the influence of $\alpha$ and $\sigma_{\max }$ was explored in our previous contributions in one-dimensional systems [18,19], and in this current paper we concentrate our study on a situation in which the clogging is expected ( $\alpha$ large and $\left.\sigma_{\max }=1\right)$ and therefore is the most important situation to be explored.

\section{RESULTS}

We start the analysis of our system of particles by studying the time evolution of mobility and $\Phi_{x}$ for considerably low stochastic level $\alpha=20$ [26] for some different values of density, here defined as $\rho=N / V$, i.e., the number of particles divided by the number of cells. Thus we run our simulations for three different values of densities: $\rho=0.5,1.0$, and 1.5. At this regime, we expect the system of dimensions $L_{x}=256$ and $L_{y}=64$ to present a high mobility scenario for low density, while for the high density case we expect the system to jam. Figures 1 and 2 show the expected behavior for the two present extreme cases of density [plots (a) and (c)] as we can see by $M(t)$ and $\Phi_{x}(t)$ for each of the ten runs rapidly reaching the steady state. The case of intermediate density, i.e., $\rho=1.0$, surprisingly shows a kind of transient instability

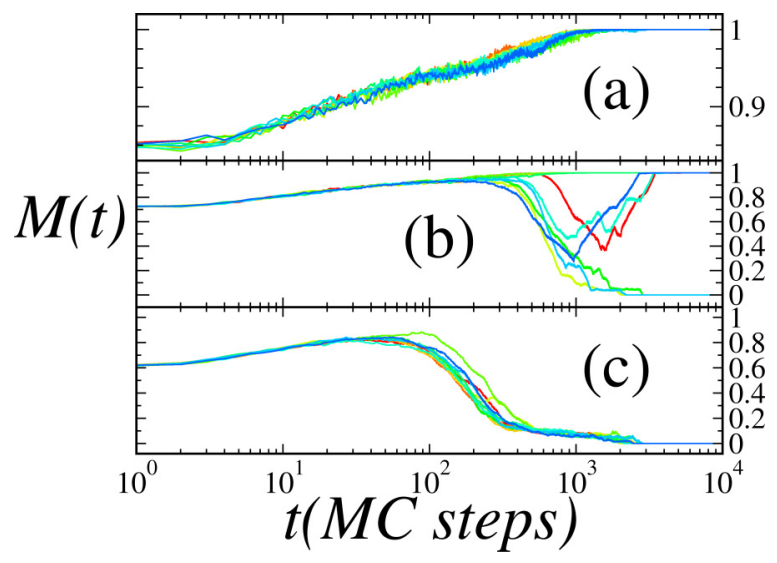

FIG. 1. Time evolution of mobility for ten different runs (different seeds, corresponding to different colors, or different shades of gray in grayscale) of a system with dimensions of $L_{x}=256$ and $L_{y}=$ 64 and with $\alpha=20$. The dynamics relaxes to a state of optimal flow when $\rho=0.5$ (a) and to an immobile state when $\rho=1.5$ (c). For $\rho=1.0$ (b), the system presents a transient instability phenomenon on the steady states where either the mobile or the immobile phase can arise depending on the time series.

phenomenon, as we can see by plot (b) in Figs. 1 and 2. The present case reveals that for a certain specific density, the steady state of each run depends sensibly on the initial conditions, as we can observe by the splitting of the mobility and the $x$-order parameter. We can also observe in plot (b) of Figs. 1 and 2 that it is a bimodal situation, since there is no intermediate steady state of mobility other than the fully mobile self-organized state (lanes) or the jammed state.

To better understand this transient instability, we studied the system dependence on the density $\rho$. By knowing that the dynamics relax to a bimodal steady state of extremely different outcomes, where for each run the mobility and $\Phi_{x}$ do not change after a sufficient time, we implemented the same criteria to stop the time evolution as used in the previous work based on the slope of the time-dependent curve to consider

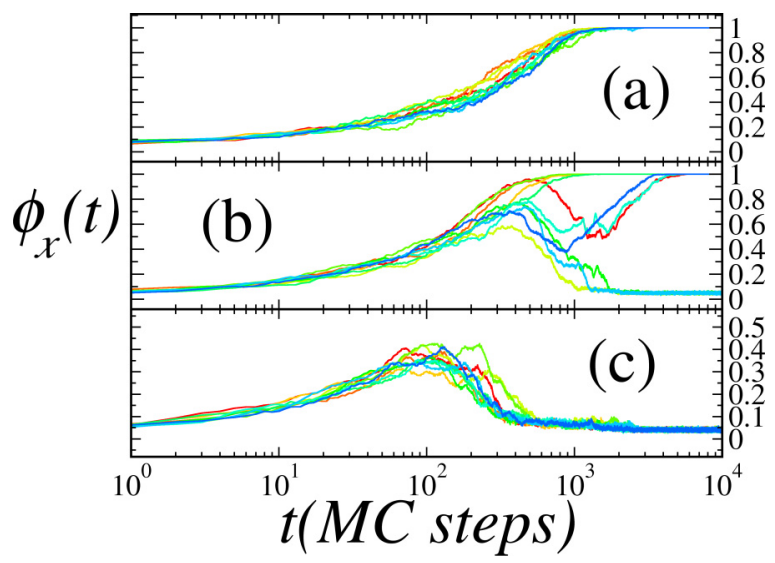

FIG. 2. Time evolution of the alternative order parameter $\Phi_{x}$ from the same set of time series plotted in Fig. 1 for the mobility. Here the conclusions are exactly the same as that obtained for the time evolution of the mobility. Different colors, or different shades of gray, correspond to different seeds. 


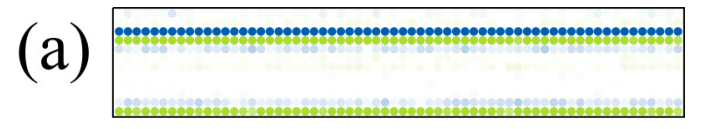

(b)

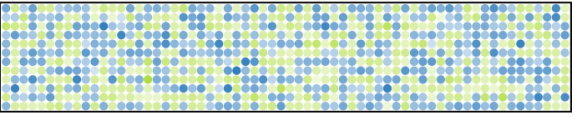

(c)

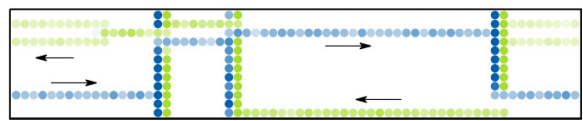

FIG. 3. Typical snapshots obtained when having high density: $\rho_{A}=\rho_{B}=8$. We can see that the system presents (a) lane patterns, (b) a clogging state, and (c) a strange situation where where clogging of particles and a lane-organized pattern seems to be simultaneously coexisting, the so called "unorganized state". Blue (dark gray) correspond to particles moving to the right side, Green (light gray) correspond to ones that are moving to the left side.

when the system has reached the steady state. The considered criteria consist of making a linear fitting on $M(t)$ and $\Phi_{x}(t)$ averaged over consecutive large number of time intervals $\Delta t$, thus comparing the slope of the fitted curves to an error value $\eta$. In this work, we used $\Delta t=1000$ and $\eta=10^{-7}$, which was shown in Ref. [18] to be a good choice. Now focusing on the study of the density's influence on the transient instability behavior, we simulated $N_{\text {run }}$ different runs to calculate the probability of lane formation (the number of times that the system reaches the lane pattern at steady state divided by $N_{\text {run }}$ ), which is denoted as $p_{\text {lane }}$. Similarly, we calculated the probability of clog formation, denoted as $p_{\text {clog }}$, and the probability of any outcome other than the two previous cases, denoted as $p_{\text {else }}=1-p_{\text {clog }}-p_{\text {lane }}$.

First, it is interesting to observe the other outcome (different from clogging and lane pattern) that occurs in our problem, i.e., what we refer to as the "else" situation. To understand this point, let us observe the snapshots of the possible different patterns shown in Fig. 3. Here we used a high particle density: $\rho_{A}=\rho_{B}=8.0$. Figure 3(a) shows the lane pattern (certainly rare in this situation), Fig. 3(b) illustrates the clogging state (highly probable in this situation), and finally in Fig. 3(c) we show the occurrence of a very different pattern of fluidity, denoted "unorganized" pattern of mobility, which simultaneously contains characteristics from states (a) and (b), i.e., a coexistence of these states.

Here it is important to clarify the apparent contradiction between plots (c) of Figs. 1 and 2 and what the snapshots show. The situation (c) plotted in Fig. 3 is indeed very rare for higher values of density in longitudinal systems and when the species are balanced $\left(\rho_{A}=\rho_{B}\right)$ in the environment, although this deserves more attention. It is so rare that when we created plot (c) of Figs. 1 and 2, all the time series sampled relaxed to the clogging steady state, which is really expected for $L_{x} \rightarrow \infty$. However, when one explores a reasonable sample, and less longitudinal systems as those used to build the snapshots $\left(L_{x}=64\right.$ and $\left.L_{y}=12\right)$, we obtain this rare mobile steady state (which in this case is not so rare even for high density systems) corresponding to plot (c) of Fig. 3. However, Figs. 1(b) and 2(b) suggest that lane patterns and clogging

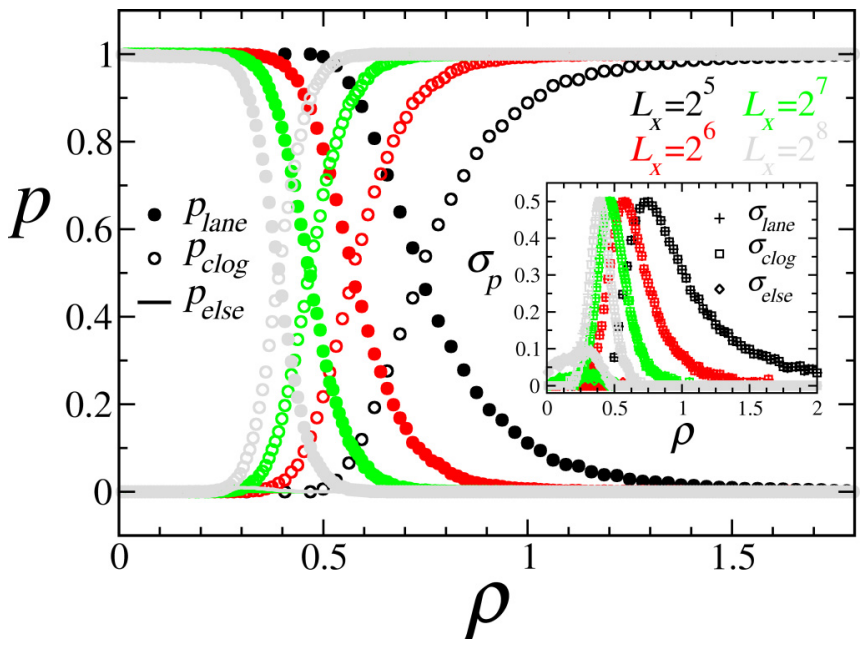

FIG. 4. The probabilities of lane formation (filled circles), jamming (empty circles), and relaxation to any other state in between (lines) for a system with a width of $L_{y}=2$ for $L_{x}=2^{5}$ (black), $L_{x}=2^{6}$ (red), $L_{x}=2^{7}$ (green), and $L_{x}=2^{8}$ (gray). We are able to see that the crossover density decreases asymptotically as the length of the system increases. The inset shows the standard deviation of the probabilities having their maximum value $\rho_{c}$ decreasing with lengthy systems. The different colors used in this figure correspond to different shades of gray in grayscale. Our simulations lead to $p_{\text {else }} \approx 0$.

can occur for the same, but not so high, density value and the question is, what kind of perturbation should be used to create the coexistence between the lane pattern and jammed states?

It is important to mention that in our model, one species only interacts with the counter (opposite) species since the particles of the same species are in the same stream, and thus this can lead to mobile systems even for higher densities. It is interesting to investigate these points further by looking at the properties of the system at steady state for a density range and making clear what such a crossover is and other nuances of this problem.

Figure 4 shows the probabilities of the system reaching each one of the three possible steady states as a function of $\rho$ for a system of width $L_{y}=2$ (purposefully quite narrow) and different values of length $L_{x}=2^{5}$ (black), $L_{x}=2^{6}$ (red), $L_{x}=2^{7}$ (green), and $L_{x}=2^{8}$ (gray). We can observe that the system shows the expected lane formation state for small densities regardless of the size of the system. When the system has sufficiently large density ( $\rho \geqslant 0.5$ for $L_{x}=2^{5}$, for example), clogging starts to emerge as a possible steady state, and the bimodal scenario arises. With larger density values, $p_{\text {clog }}$ keeps increasing while $p_{\text {lane }}$ decreases until both curves cross each other over and the most probable scenario for the dynamics shifts.

The inset of Fig. 4 shows us that the standard deviation of $p$ has its maximum value at the crossover density where $p_{\text {lane }}=$ $p_{\text {clog }}=1 / 2$ and thus we define the crossover density $\rho_{c} \equiv$ $\rho\left(p_{\text {lane }}=p_{\text {clog }}=1 / 2\right)$. As Fig. 4 suggests, the lengthier the system is, the more the crossover density appears to decrease asymptotically in conjunction with the range of density.

Let us continue with our finite-size scaling study, but now changing the width of a long system. We show in Fig. 5 the 


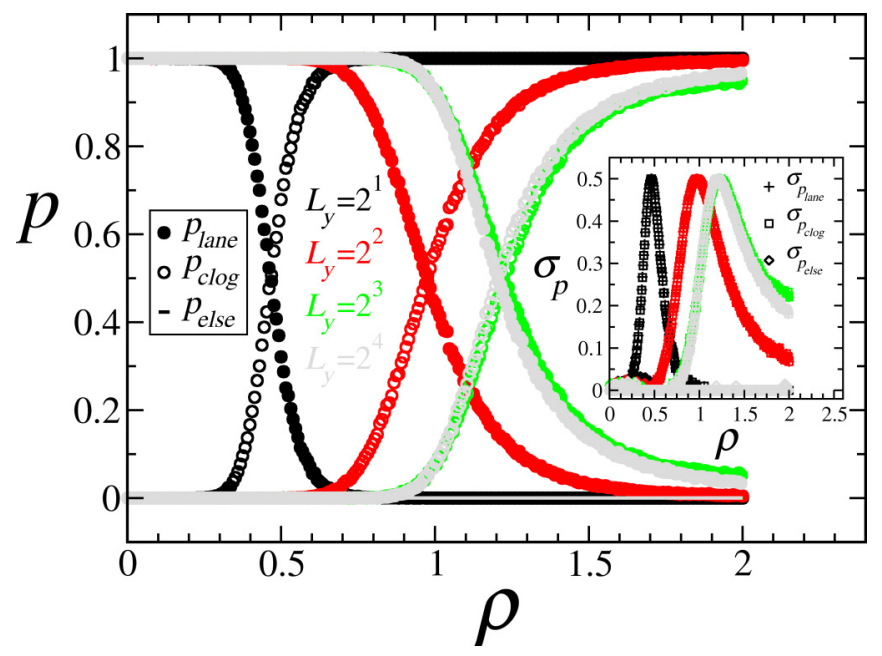

FIG. 5. The probabilities of lane formation (filled circles), jamming (empty circles), and relaxation to any other state (lines) for a system of length $L_{x}=128$. We plotted the results for different widths: $L_{y}=2$ (black), $L_{y}=2^{2}$ (red), $L_{y}=2^{3}$ (green), and $L_{y}=2^{4}$ (gray). We notice that the crossover density increases from $\rho_{c} \approx 0.5$ up to a maximum $\rho_{c} \approx 1.2$ for systems with $L_{y} \geqslant 2$. The different colors used in this figure correspond to different shades of gray in grayscale. Our simulations lead to $p_{\text {else }} \approx 0$.

probabilities of the stationary outcome for different widths $L_{y}=2$ (black), $L_{y}=2^{2}$ (red), $L_{y}=2^{3}$ (green), and $L_{y}=2^{4}$ (gray) while keeping the length fixed on $L_{x}=128$. We observe that when the toroid is considerably narrow, the system exhibits a large difference in $\rho_{c}$, as we can see that it increases for the smaller widths $L_{y}=2,2^{2}$, and $2^{3}$. However, when the system has a width of $L_{y}=2^{3}$ or greater, the crossover density does not change and we would expect the chances of lane formation to increase with wider corridors, but this does not occur due to the proportionally larger number of particles.

Thus by denoting the concentration of the species as $c_{A}=$ $N_{A} / N$ and $c_{B}=N_{B} / N$ and remembering that we used $c_{A}=c_{B}$ so far, we see from Figs. 4 and 5 that $p_{\text {else }} \approx 0$, showing that unorganized mobility patterns are very rare in this situation. Similarly, for higher densities, lane patterns also rarely occur since $p_{\text {lane }} \approx p_{\text {else }} \approx 0$.

We also approached the problem considering different relative concentration of particles, i.e., different mixing of species. Figure 6 shows the stationary state of the dynamics when the proportion of the species ranges from a scenario of basically one species moving along the toroid $\left(c_{A}=0\right.$, where $\left.c_{A}+c_{B}=\frac{N_{A}+N_{B}}{N}=1\right)$ to the case studied until this point of equal concentrations $c_{A}=c_{B}=1 / 2$. In the present plot (Fig. 6), we show the three regimes that arise when $\rho<\rho_{c}$, $\rho=\rho_{c}$, and $\rho>\rho_{c}$. For a system with dimensions $L_{x}=128$ and $L_{y}=16$, the crossover density is $\rho_{c} \approx 1.2$ (light gray curve in Fig. 5), and we therefore simulated the cases $\rho=0.5$, 1.2, and 3.0, which are shown in the plots of Figs. 6(a), 6(b), and $6(\mathrm{c})$, respectively.

For all the curves, we began by simulating a scenario of one species $\left(c_{A}=0\right)$, which means $p_{\text {lane }}=1$ and obviously it represents a fake state of lane formation because there is no induced self-organization when only one species is considered.

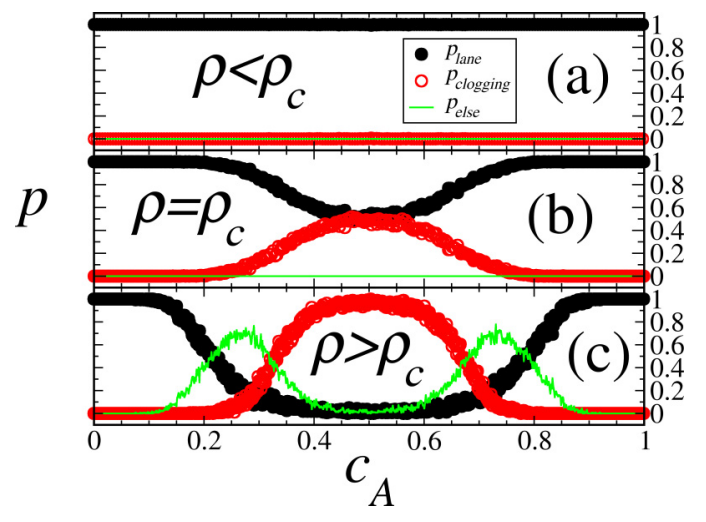

FIG. 6. The dependence of the probabilities ( $p_{\text {lane }}, p_{\text {clog }}$, and $p_{\text {else }}$ ) on the relative density for three values of the absolute density. For $\rho<\rho_{c}$ (a) the system will flow regardless of the mixing of species. For $\rho=\rho_{c}$ (b) the system has $p_{\text {lane }}=1$ for $c_{A} \leqslant 0.2$ and falls to $p_{\text {lane }}=0.5$ when the mixing increases up to a $1: 1$ ratio, reaching the point where we defined the crossover density. Finally, when $\rho>\rho_{c}$ (c) the system is more likely to present the steady state of phase coexistence in the interval of $0.1<c<0.4$.

Figure 6(a) shows an expected behavior for the dynamics because the average level of occupation $(\rho)$ of the system ensures the lane formation despite the growth of the concentration of species $A$. However, the case of $\rho=\rho_{c}$ [Fig. 6(b)] shows that $p_{\text {lane }}$ gradually decreases as $c_{A}$ grows. Finally, in Fig. 6(c), which corresponds to $\rho>\rho_{c}$, we observe that the system can relax to a situation in which it is neither only self-organized by lanes nor only relaxed to a jammed state (coexistence). In that case, the overall density $\rho$ is above the crossover level, so the growth of $c_{A}$ around $c_{A} \approx 0.2$ makes the particles $A$ stand as impurities for the movement of $B$, but they are not sufficient to make the system immobile. When the mixing of species tends to the same value $\left(c_{A} \approx\right.$ $c_{B}$ ), the system essentially shows the clogging steady state uniquely.

To conclude, it is important to make some points about $\alpha$. We used a sufficiently large value of $\alpha$ throughout our paper, but this was not duly justified. To do so, we performed simulations to obtain a plot of $p \times \alpha$ considering the same sized system $\left(L_{x}=128\right.$ and $\left.L_{y}=16\right)$, keeping $c_{A}=c_{B}$, which was used for most of this paper, and using $\rho=0.5<\rho_{c}$, $\rho=\rho_{c} \approx 1.2$, and $\rho=3.0>\rho_{c}$, as shown in plots (a), (b), and (c), respectively, of Fig. 7 .

The result shows that the dynamics has an abrupt transition on the stochastic level $\alpha$, and that abrupt transition depends on the density $(\rho)$, similar to what was observed in the original one-dimensional model $[18,19]$. The result also suggests that for $\rho=\rho_{c}$, the system is more likely to jam in the range $2 \lesssim \alpha \lesssim 5$, where the particle interaction is sufficiently high as to make the system relax to an immobile state but not to self-organize in lane patterns. For small values of $\alpha$, the system is moderately mobile, which is expected since the interaction between opposite species is weak, and particles perform biased random walks along the $x$-direction with diffusive (standard random walks) components in the $y$ direction. For high values of $\alpha$, the system shows the expected equally probable steady states of mobile lane patterns and jamming. 


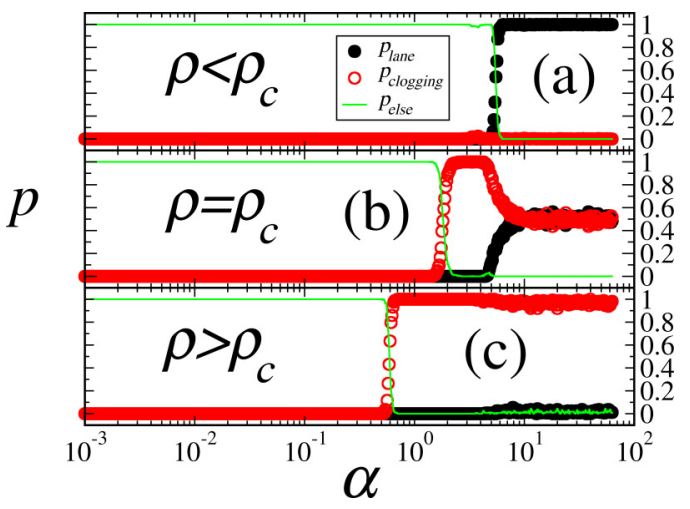

FIG. 7. Behavior of $p$ as a function of $\alpha$ considering $c_{A}=c_{B}$ for the same size and other parameters used in Fig. 6. For $\rho=\rho_{c}$, the system shows an intermediate range $(2 \lesssim \alpha \lesssim 5)$, where the system is more likely to jam.

\section{SUMMARIES, CONCLUSIONS, AND DISCUSSIONS}

In this paper, we extend the Fermi-like model for particles in counterflowing streams to a two-dimensional lattice, and we show the existence of three possible steady states for the system: (i) a lane-organized state, (ii) a jamming/clogging state, and (iii) an "unorganized" state (metastable) where a jammed cluster of particles (clogs) and mobile lane patterns can coexist for a highly dense system $\left(\rho>\rho_{c}\right)$. This occurs when one breaks the balance/symmetry, i.e., when $c_{A} \neq c_{B}$. We can observe that such coexistence (although unlikely because the system should evolve to a state of condensation of particles in this situation) can occur even for small perturbations around $c_{A}=c_{B}=1 / 2$, as suggested by the fluctuations observed for $p_{\text {else }}$ in Fig. 6.
It is interesting that in the context of driven lattice gases [27] and cellular automata for traffic flow [28], similar metastability phenomena can be observed as well as the presence of a phase-separated steady state of coexistence between a jammed and a mobile phase, and it is also interesting to observe how such peculiarity occurs in counterflowing streams of particles.

Our results also suggest that a larger imbalance/asymmetry between the species makes the three possible states likely to happen. In this situation, $p_{\text {else }}$ reaches its maximum value at $c_{A} \approx 1 / 4$ and symmetrically at $c_{A} \approx 3 / 4$ for $\rho>\rho_{c}$ (see Fig. 6). On the other hand, $p_{\text {else }}=0$ in more diluted situations: $\rho \leqslant \rho_{c}$ independently of $c_{A}$, showing the absence of coexistence in such a situation. The coexistence also vanishes for flows of a single species ( $c_{A}=0$ or 1$)$ for any density.

Last but not least, we observe the effects of the randomness parameter $\alpha$ according to Fig. 7. Our results still suggest that for sufficiently large values of $\alpha$, one observes that for $\rho=\rho_{c}$, $p_{\text {lane }}=p_{\text {clog }} \approx 1 / 2$, which is the expected value according to our definition. Finally, for small values of $\alpha$, the system is flowing under unorganized patterns since $p_{\text {else }}=1$ is a result from the biased random-walk dynamics.

\section{ACKNOWLEDGMENTS}

This work was partly developed using the computational resources from Cluster Ada, IF-UFRGS. R.d.S. and E.V.S. wish to thank $\mathrm{CNPq}$ for partly supporting this work under Grants No. 311236/2018-9, No. 424052/2018-0, and No. $154822 / 2016-7$. In addition, the authors would like also to thank the anonymous referees for calling our attention to the existence of metastability in some similar systems in statistical mechanics.
[1] B. Schmittmann and R. K. P. Zia, Statistical mechanics of driven diffusive systems, in Phase Transitions and Critical Phenomena, edited by C. Domb and J. Lebowitz (Academic, London, 1995), Vol. 17, pp. 3-214.

[2] B. Schmittmann, K. Hwang, and R. K. P. Zia, Europhys. Lett. 19, 19 (1992).

[3] R. da Silva, A. Hentz, and A. Alves, J. Phys. A 437, 139 (2015).

[4] C. L. N. Oliveira, A. P. Vieira, D. Helbing, J. S. Andrade, Jr., and H. J. Herrmann, Phys. Rev. X 6, 011003 (2016).

[5] E. V. Stock, R. da Silva, and H. A. Fernandes, Phys. Rev. E 96, 012155 (2017).

[6] Y. Tajima, K. Takimoto, and T. Nagatani, J. Phys. A 313, 709 (2002).

[7] J. Dzubiella, G. P. Hoffmann, and H. Lowen, Phys. Rev. E 65, 021402 (2002).

[8] M. Rex and H. Löwen, Phys. Rev. E 75, 051402 (2007).

[9] T. Vissers, A. van Blaaderen, and A. Imhof, Phys. Rev. Lett. 106, 228303 (2011).

[10] T. Vissers, A. Wysocki, M. Rex, H. Lowen, C. P. Royall, A. Imhof, and A. van Blaaderen, Soft Matter 7, 2352 (2011).

[11] I. Buttinoni, J. Bialke, F. Kummel, H. Lowen, C. Bechinger, and T. Speck, Phys. Rev. Lett. 110, 238301 (2013).
[12] T. Kretz, A. Grunebohm, M. Kaufman, F. Mazur, and M. Schreckenberg, J. Stat. Mech. (2006) P10001.

[13] M. Isobe, T. Adachi, and T. Nagatani, J. Phys. A 336, 638 (2004).

[14] D. Helbing and P. Molnar, Phys. Rev. E 51, 4282 (1995).

[15] W. Guo, X. Wang, and X. Zheng, J. Phys. A 432, 87 (2015).

[16] S. Nowak and A. Schadschneider, Phys. Rev. E 85, 066128 (2012).

[17] In this paper, we use the terms "clogging" and "jamming" interchangeably, even knowing that they are not exactly synonymous. We understand that other authors also use these terms indiscriminately.

[18] R. da Silva and E. V. Stock, Phys. Rev. E 99, 042148 (2019).

[19] E. V. Stock, R. da Silva, and C. R. da Cunha, J. Stat. Mech. (2019) 083208.

[20] S. N. Majumdar, M. R. Evans, and R. K. P. Zia, Phys. Rev. Lett. 94, 180601 (2005).

[21] E. W. Montroll and B. West, On Enriched Collection of Stochastic Process: In Fluctuation Phenomena, edited by E. W. Montroll and J. Lebowitz (Elsevier, Amsterdam, 1979).

[22] H. Ohta, Europhys. Lett. 99, 40006 (2012).

[23] A. I. Curatolo, M. R. Evans, Y. Kafri, and J. Tailleur, J. Phys. A 49, 095601 (2016). 
[24] R. Dickman, Phys. Rev. A 38, 2588 (1988).

[25] R. Dickman, Phys. Rev. E 64, 016124 (2001).

[26] For $\alpha=20$, the system is essentially deterministic, which is exactly our purpose since only in this regime can the coexistence of states occur. For this reason, we concentrate a discussion about $\alpha$ in the final part of this paper, presenting a plot to justify this initial choice of our study.

[27] C. Appert and L. Santen, Phys. Rev. Lett. 86, 2498 (2001).

[28] R. Barlovic, L. Santen, A. Schadschneider, and M. Schreckenberg, Eur. Phys. J. B 5, 793 (1998). 